\title{
Deformation quantization of Noncommutative Principal Bundles
}

\section{Paolo Aschieri*}

Dipartimento di Scienze e Innovazione Tecnologica, Univ. del Piemonte Orientale

E-mail: aschieriato.infn.it

Drinfeld twist deformation theory of modules and algebras that carry a representation of a Hopf Algebra $H$ can be extended to deform also morphisms and connections that are not $H$-equivariant. In this talk I present how similar techniques allow to canonically deform principal $G$-bundles, and in general how Hopf-Galois extensions are canonically deformed to new Hopf-Galois extensions. Twisting the structure group we obtain principal bundles with noncommutative fiber and where the structure group is a quantum group. Twisting the automorphism group of the principal bundle we further obtain a noncommutative base space.

Frontiers of Fundamental Physics 14 - FFP14,

15-18 July 2014

Aix Marseille University (AMU) Saint-Charles Campus, Marseille

\footnotetext{
${ }^{*}$ Speaker.
} 